$\mathrm{CO}_{2}$-Enriched

\section{Atmosphere on the Microscope Stage}

BioTechniques 27:292-294 (August 1999)

A large number of cell structures can now be detected and monitored in situ using fluorescent molecular probes (4). The use of supravital probes is rather easy, but requires the maintenance of good viability conditions for the whole duration of experiments. While there is general concern about the control of the medium temperature, by means of hot air curtain or resistance-thermostated stages, little information is provided in literature about the maintenance of a stable physiological $\mathrm{pH}$, particularly when the medium is buffered with sodium bicarbonate (>2 g/L $\left.\mathrm{NaHCO}_{3}\right)$. This salt, dissolved in water, tends spontaneously to release $\mathrm{CO}_{2}$ in the atmosphere $\left(\mathrm{NaHCO}_{3} \Leftrightarrow \mathrm{NaOH}+\mathrm{CO}_{2}\right)$ with a progressive $\mathrm{pH}$ increase $(1,2)$. For this reason, $\mathrm{NaHCO}_{3}$-buffered media must be kept under a $\mathrm{CO}_{2}$-enriched atmosphere (usually $4 \%-6 \% \mathrm{CO}_{2}$ ) to prevent medium alkalinization. As soon as the medium is taken from the $\mathrm{CO}_{2}$ thermostat, the low air $\mathrm{CO}_{2}$ content $(0.3 \%-0.4 \%)$ causes a rise of the medi-

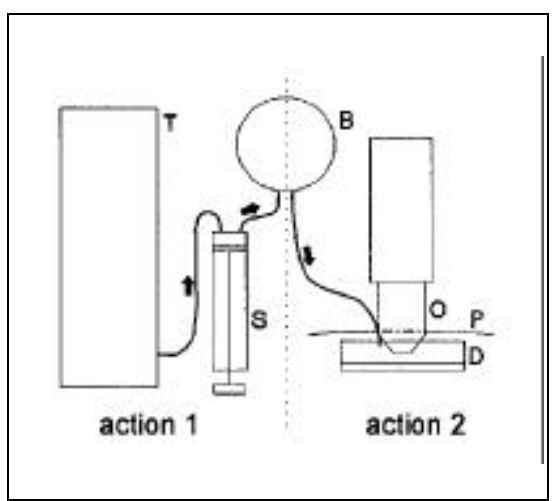

Figure 1. $\mathrm{CO}_{2}$-enriched air atmosphere on the microscope stage. The gas is taken from the $\mathrm{CO}_{2}$ thermostat outlet ( $\mathrm{T}$ ) and pumped into a balloon (B) by means of a large syringe (S) provided with two check valves. The balloon is then connected to a tube, ending with a short tip of $300 \mu \mathrm{m}$ of diameter that is fixed to the microscope objective (O) and directed to the culture dish (D). A loose dish cover is made by a flange of Parafilm (P) fitted to the objective. The gas flow maintains the medium $\mathrm{pH}$ at the original value found in the thermostat. um $\mathrm{pH}$, well out of the physiological range. Gas-tight chambers, which may be useful in specific situations and adapted to particular instruments (5), are not suitable for operations with upright fluorescence microscopes. An alternative is that of transferring cells into media buffered with phosphate or 4-(2hydroxyethyl)-1-piperazineethanesulfonic acid (HEPES), which do not require $\mathrm{CO}_{2}$ equilibration. But in this case, the abrupt medium change might disturb or damage cells. Moreover, HEPES buffer is not ideal for these studies because its exposure to light generates cytotoxic products $(3,6)$. In this report, we propose a simple method to create a $\mathrm{CO}_{2}$-enriched atmosphere on the microscope stage. The gas is directly aspired by a 1000-cc syringe (Hamilton, Reno, NV, USA) from the $\mathrm{CO}_{2}$ thermostat through the built-in outlet used for the $\mathrm{CO}_{2}$ measurement and pumped into a balloon. Unfortunately, balloons are not commercially available as technical or laboratory products. However, balloons of the kind used by children work well, provided that they are previously ventilated to exclude any trace of volatile plasticizers. Both actions (gas suction and pumping) can be done in sequence if the syringe is provided with two check valves: one for the input (thermostat $\rightarrow$ syringe) and one for the output

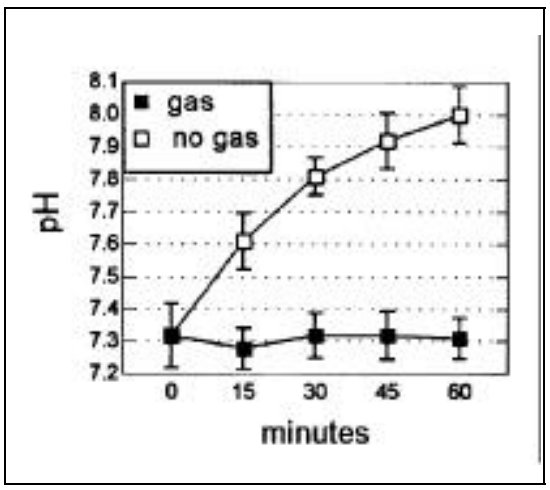

Figure 2. pH changes in DMEM buffered with $3.7 \mathrm{~g} / \mathrm{L} \mathrm{NaHCO}_{3}$ with and without $5 \% \mathrm{CO}_{2}$ air perfusion. Without gas perfusion, a substantial increase of $0.3 \mathrm{pH}$ units is evident as early as $15 \mathrm{~min}$ after air exposure. After $30 \mathrm{~min}$, the $\mathrm{pH}$ of the air-exposed medium is 7.8 , out of the socalled physiological range, and continues to increase at later times. The perfused medium is stable for the whole time interval tested. Gas perfusion is obtained as described in Figure 1. pH-meter sensitivity: $0.01 \mathrm{pH}$ units. (syringe $\rightarrow$ balloon). The $\mathrm{CO}_{2}$-air mixture is quickly renewed in the thermostat. The balloon is then connected to a generic tube, ending with a 4-5-cmlong polyethylene tube of $300 \mu \mathrm{m}$ of diameter (a catheter). This end is fixed to the microscope objective with the tip directed to the medium surface. The tip is not immersed in the medium, so that the gas flow does not perturb the medium surface. To increase the retention of the gas, a flange of Parafilm ${ }^{\circledR} \mathrm{M}$ (American National Can, Norwalk, CT, USA) can be fitted to the objective to form a loose dish cover. The system, shown in Figure 1, produces a gas flow of approximately $100 \mu \mathrm{L} / \mathrm{s}$ for $2-3 \mathrm{~h}$. In case of longer sessions or accidental rupture, the balloon can be replaced in the turn of few seconds. In this way, the medium $\mathrm{pH}$ is stable for long time intervals. Figure 2 shows the effect of a $5 \% \mathrm{CO}_{2}$-air mixture on the $\mathrm{pH}$ of $2 \mathrm{~mL}$ of Dulbecco's modified Eagle medium (DMEM) (Life Technologies, Gaithersburg, MD, USA) with $3.7 \mathrm{~g} / \mathrm{L} \mathrm{Na-}$ $\mathrm{HCO}_{3}$. After $1 \mathrm{~h}$ the $\mathrm{pH}$ is unchanged in the perfused medium, whereas it is 0.7 units higher in the air-exposed medium. However, as early as after $15 \mathrm{~min}$, the air-exposed medium $\mathrm{pH}$ is 0.3 units over the initial value. Because an equivalent time interval is normally spent for preliminary operations (to place the sample on the stage, adjust optics, set the camera, search fields, etc.) and subsequent observations before the last image is acquired, the risk of experimental bias due to the progressive $\mathrm{pH}$ alteration subsists in the majority of cases in which cells in high $\mathrm{NaHCO}_{3}$-buffered media are to be observed in vivo.

\section{REFERENCES}

1.Adams, R.L.P. 1990. Cell culture for biochemists, p. 35-38. In R.H. Burdon and P.H. Van Knippenberg (Eds.), Laboratory Techniques in Biochemistry and Molecular Biology. Vol. 8, 2nd ed. Elsevier, Amsterdam.

2.Freshney, R.I. 1986. Animal Cell Culture. A Practical Approach, p. 39-41. IRL Press, Oxford.

3.Lepe-Zuniga, J.L., J.S. Zigler, Jr. and I. Gery. 1987. Toxicity of light-exposed Hepes media. J. Immunol. Methods 103:145.

4.Mason, W.T. (Ed.). 1993. Fluorescent and Luminescent Probes for Biological Activity. Academic Press, London.

5.Rotsch, C., K. Jacobson and M. Radmacher. 1999. Dimensional and mechanical 
dynamics of active and stable edges in motile fibroblasts investigated by using atomic force microscopy. Proc. Natl. Acad. Sci. USA 96:921-926.

6.Spierenburg, G.T., F.T. Oerlemans, J.P. vanLaarhoven and C.H. de Bryun. 1984. Phototoxicity of N-2-hydroxyethylpiperazine- $\mathrm{N}^{\prime}$ 2-ethanesulfonic acid-buffered culture media for human leukemic cell lines. Cancer Res. $44: 2253-2254$

Address correspondence to Dr. Giacomo Diaz, Dipartimento di Citomorfologia, Università di Cagliari ,Cittadella Universitaria di Monserrato, 09042 Monserrato (CA), Italy.Internet: gdiaz@unica.it

Received 8 January 1999; accepted 3 May 1999.

\section{Giacomo Diaz, Raffaella Isola, Angela Maria Falchi and Andrea Diana University of Cagliari Cagliari, Italy}

\section{Polyacrylamide Gel Fixation by Drying and Gluing to Both Paper and Transparent Plastic}

BioTechniques 27: 294 (August 1999)

Even in the age of computer scanners and imagers, perfect gel fixation still remains a problem. The described method was designed to obtain permanent records of the polyacrylamide gels for visual inspection. The advantages of the present protocol (see Table 1) are: (i) the avoidance of expenses, because it requires neither special apparatus nor expensive reagents; and (ii) the protection of the dried gel with the transparent plastic. The resulting image, in our opinion, is much more durable than those prepared by any other methods (1-3), and, in our experience, it is not subject to occasional damage. We use the following materials: (i) $3 \mathrm{MM}$ paper (Whatman, Clifton, NJ, USA) or an

Table 1. Protocol

1. Proceed with the electrophoresis, staining and destaining of the gel to be dried. The protocol of staining and destaining seems not to be critical for the present method of drying. We use the most conventional one:

Staining the gel for $2 \mathrm{~h}$ with $0.1 \%$ Coomassie ${ }^{\circledR}$ Blue R-250 in

fixative (40\% methanol, $10 \%$ acetic acid).

Destaining the gel with several changes of the same fixative to remove background (usually for 6-12 h).

2. Trim the plastic sheet and Whatman paper to be within 1-2 cm larger than the gel on each side.

3. Place the gel to the middle of the damp Whatman paper. Remove the bubbles, and place all this sandwich to the stack of paper towels 2-3-cm high. Let it dry for 15-20 min. The water solution on the upper surface of the gel should evaporate. Do not let the corners of the gel curve.

4. Smear the gel with the glue $\left(0.05-0.1 \mathrm{~mL} / \mathrm{cm}^{2}\right)$. Be sure that the entire surface, especially the edges, is well covered.

5. Put the plastic sheet onto the glue layer. Try to avoid the formation of big bubbles under the plastic surface. Bubbles 1-2 mm in diameter are not critical.

6. Place a 5-10-kg weight with a flat bottom to cover the entire surface of the gel. Allow drying of the gel to proceed for $12-24 \mathrm{~h}$. If possible, replace the towels periodically.

7. When drying is completed (the glue layer becomes absolutely transparent), trim the plastic and the Whatman paper around the gel perimeter. Adhere the resulting picture to the working diary with the adhesive tape or with the same glue.

analogous, (ii) transparent plastic-the source is not critical. We routinely use the Verbatium ${ }^{\mathrm{TM}}$ laser printer transparencies (Verbatim, Charlotte, NC, USA) and (iii) polyvinylacetate glue ("white glue"; Akron, Novgorod, Russia) or the Elmer's ${ }^{\circledR}$ Glue-All (Borden, Columbus, $\mathrm{OH}, \mathrm{USA}$ ).

The process described in Table 1 is similar to Southern transfer. The destaining solution migrates out of the gel to the paper towels, being gradually replaced by the glue. The pre-polymer component of the glue migrates no further than Whatman paper, thus adhering the dried gel to both paper and plastic. Even the subsequent cracking of the dried gel does not influence the quality of the resulting image. After drying, the visual detection of some protein bands is enhanced because the background is white and the protein bands become darker. The gels do not shrink after this drying, except for a slight deformation on the edges. Owing to the usage of the glue, any kind of transparent plastic, which can ensure the desirable durability of the resulting image, can be used in this system.

\section{REFERENCES}

1.Michaels, S.D. and J.C. Ford. 1991. Inexpensive method for air-drying polyacrylamide electrophoresis gels. BioTechniques 11:466467.

2.Porter D.R. and M.J. Gatscef. 1992. Simplified drying of polyacrylamide gels for fluorography. BioTechniques 13:364-365.

3.Smith, B.J. 1994. Drying gels. Methods Mol. Biol. 32:157-161.

Address correspondence to Yuri V. Guervaziev, Lab. of Molecular Biotechnology, Institute of Biomedical Chemistry, Pogodinskaya Str., 10, 119832, Moscow, Russia. Internet: yura@medic.ibmh.msk.su

Received 2 November 1998; accepted 18 May 1999.

\section{Yuri V. Guervaziev \\ Institute of Biomedical \\ Chemistry \\ Moscow, Russia}

